\title{
The Evolution of PET/MR Is Hindered by Our Field's Reluctance to Provide Critical Evaluation
}

\author{
Adam L. Kesner
}

Memorial Sloan Kettering Cancer Center, New York, New York

$\mathbf{O}$

ver the last 10 years, our field has been able to review and develop opportunities for integrating commercially available PET/ MR into clinical practice. We have done so with a coalition of industry, academia, and health-care providers. Although the intention to deliver better technology to patients through dissemination of PET/MR is laudable, the delineation between academic and clinical utility is often omitted from the literature; this impedes the field in finding the proper context for the technology. Under this circumstance, I am writing to follow up on a recent article published in The Journal of Nuclear Medicine: "PET/MRI Versus PET/CT for Whole-Body Staging: Results from a SingleCenter Observational Study on 1,003 Sequential Examinations" by Martin et al. (1). The work that is described in this publication portrays a benchmark comparison of new and classic technologies relative to the classic domain of oncology in PET, but there are notable biases in the study design worthy of comment. Furthermore, the publication presents an opportunity to remark on many of the practical differences between PET/MR and PET/CT, which when presented alongside the authors' analysis, could portray a case for arriving at an opposite conclusion. Ultimately, 10 years into the commercial PET/MR era, the field has yet to contextualize the technology in academic and clinical-value equations, which promotes exclusivity and hinders opportunities to identify and address the barriers that encumber clinical impact.

The work performed by Martin et al. compares diagnostic capacities between an implementation of a PET/CT system and an implementation of a PET/MR system in a given population. However, study design differences across cameras were numerous and uniformly biased in favor of the perceived superior performance of the PET/MR system. The largest bias stems from the differences in PET hardware in the systems being compared, that is, a Siemens Biograph mCT PET/CT and a Siemens mMR PET/ MR. The specifications and a performance comparison of these same, or similar, model systems have recently been reported (2). The 2 scanners have differences in bore size, transaxial coverage, crystal-to-detector encoding ratio, and photon amplifier technologies (avalanche photo diode vs. photomultiplier tubes). All these design differences favor the PET scanner associated with the mMR model (better sensitivity, better resolution, better noise discrimination) and are unrelated to the MR component of the

Received Nov. 25, 2020; revision accepted Jan. 3, 2021.

For correspondence or reprints contact: Adam L. Kesner, Memorial Sloan Kettering Cancer Center, 1250 First Ave., Room S-1119E (Box 84), New York, NY 10065

E-mail: kesnera@mskcc.org

Published online Jan. 15, 2021.

COPYRIGHT (c) 2021 by the Society of Nuclear Medicine and Molecular Imaging. DOI: 10.2967/jnumed.120.260950 instrument. It is therefore no surprise that the authors see improved diagnostic image quality on the mMR instrument. With respect to the study design: although not all details have been provided, we can deduce that PET image quality was biased toward PET/MR because the acquisition time per bed position was twice as long as that for the PET/CT device and the acquisition took place less than one isotope half-life later; that is, the PET images in the PET/MR acquisitions were generated with more count statistics and fewer random-coincidence detections. This difference is compounded by the fact that different postreconstruction filters were used between the scanners, and physiologic differences favor increased sensitivity in the later PET/MR images (as noted by the authors). It is also worth remark that the mCT system performance appears dumbed down by not using the timeof-flight reconstruction capacity of the system, which is readily available to users and has been shown to offer notable imagequality enhancement on the PET/CT device (2).

Ultimately, it is not clear if the improved lesion detectability reported by Martin et al. stemmed from having readily accessible simultaneously acquired MR images available to clinicians or from having better PET data from the incrementally superior PET hardware in the mMR machine. The distinction is quite important to many readers because it directly impacts the answer to a principal question: is PET/MR worth the additional costs and complexity of operation when compared alongside PET/CT?

An objective comparison of the 2 technologies with different overheads and workflows is challenging. It is a reasonable contention that such comparison research is laying the groundwork of benchmarking the technology to support further developments, and such benchmarking fits within an academic context aligned with a vision of continued expansion. This sentiment is portrayed by the authors' concluding that their work will "... hopefully further pave the way toward a widespread introduction of PET/MRI into clinical patient care." However, this analysis is at odds with the clinical-value context. Clinically speaking, consideration of the large gap of approximately $5 \%$ of PET/CT patients for whom the authors were not able to obtain PET/MR images because they were subject to "abortion by patient" or negated by "technical problems of PET/ MRI" is perplexingly omitted from the analysis and at odds with the authors' conclusions on the equivalence of the devices. Furthermore, from an operational perspective, comparable performance, relative to complexities and the cost of PET/MR technology, portray a case for its limited capacity and removal from the market as a PET/CT alternative, that is, the antithesis of the authors' concluding statement. These discrepancies reveal a discordance in PET/MR academic and clinical paradigms that is prevalent in the field. 
Current outperformance applications of PET/MR versus PET/ CT have not yet made their way into the clinic. Having soft-tissue anatomic imaging and the myriad of potential MR-supported functions available alongside PET may provide quantifiable benefits such as those presented by Martin et al. However, it is unclear whether those same benefits could be achieved with more cost-effective separate PET/CT and MR machines and more aggressive lower-dose PET/CT protocols (i.e., improved image reconstruction or extending PET/CT scan times to those encountered in PET/MR) or simply supernumerary imaging orders.

A complete comparison of PET/MR and PET/CT in oncologic imaging should include economic and practical considerations involved in owning and operating the technologies-relevant for virtually all potential users. PET/MR scanners have considerably higher purchase and maintenance costs (approximately 2- to 3fold), which do not currently translate into a commensurate improvement in diagnostic quality. There are additional higher costs associated with PET/MR scanner resources, maintenance, facility safety, and technologist staff and training. PET/MR generally has lower throughput than PET/CT. Paradoxically, generally speaking, the more that existing or potential protocols utilize the advantages afforded by MR on the PET/MR system, the longer the required scan acquisition times. This lower throughput further stresses economics, patient comfort, and ultimate feasibility at the patient level. Exacerbating the issue of long scan times affecting patient comfort are the loud noises that the PET/MR imposes on the patient, as well as the increased incidence of claustrophobia from smaller bores.

From an operational perspective, the MR portion of PET/MR is more complex than CT and more difficult to standardize across patients, centers, and vendors (3). PET/MR is capable of a large abundance of image acquisition techniques and protocols. However, it is more complex to operate, whereas the contrasting CT portion of a PET/CT machine is almost a 1-click operation. Furthermore, replacing the traditional $\mathrm{CT}$ technology with MR is not a 1-to-1 equivalent substitution but comes with technologic tradeoffs. The most immediate required function of having a coregistered modality acquired with PET - attenuation correction-is performed most elegantly and robustly with transmission photon imaging, that is, CT. Although the PET/MR field has largely addressed this issue, simple tasks, such as imaging research phantoms, remain challenging on contemporary commercial systems. A robust review of clinical considerations, alongside a discussion of PET/MR avenues for innovation, can be found in the literature (4).

Ultimately, assessing the value of PET/MR requires consideration across 2 paradigms: PET/MR as a research tool, and PET/ MR as a clinical support instrument in 21 st century medicine. Efforts to delineate the two are challenging because they overlap by design and by nature-uniquely, this tool received regulatory approval and was brought to the market before its clinical indications have been established. The 2 enterprises have different motivations, resources, and barometers of success that we need to contextualize if we are to deliver the technology to its most appropriate use. For example, we do not want to stifle the ambitious or improbable aims of research with day-to-day practical limitations that can be addressed later, nor, alternatively, do we want to push Cadillac technology into hospitals and health-care systems where it does not fit.

We can perhaps understand the relationship of PET/MR technology in our field from the perspective of innovation science. The label early adopters has aptly been used to self-describe current users of PET/MR technology (3). This term is derived from diffusion of innovation theory, work by Everett Rogers that was seminal in the field. It describes a role that early customers play in the potential transition of innovation from invention to widespread adoption (5). Notably, the work, and its derivatives, identify how the relationship between industry and early adopters can be synergistic with often overlapping interests-early adopters may be less sensitive to product limitations, less sensitive to price, and prone to confirmation bias. It is also worth mentioning that early adopters exist in both successful and unsuccessful innovation paradigms.

Our field has the opportunity to evaluate an innovative technology, PET/MR, and usher it into its ideal role. Through their vision of PET/MR relevance and expansion, vendors and early adopters continue to play an essential role in directing resources and energy toward cultivating potential into value. In this case, PET/MR pioneers are, by means of ownership, selfselectively less equipped to consider challenges of scalable and cost-efficient delivery of medicine. The greater academic field must partner and play an essential role providing robust perspective and evaluation, including due critical analysis. In between the vision for innovation and its impact lie the shortcomings of the vision that need to be addressed. Patients, health-care providers, vendors, and academic communities all stand to benefit from finding a pathway for the expansion of PET/MR beyond small academic clusters to larger communities or to understand if there is not a reasonable one.

We are 10 years into the age of commercial PET/MR, and the role of the technology remains indeterminant. Academic scientists, physicians, and journals such as The Journal of Nuclear Medicine play roles in supporting the development of both research and clinical standards. Ultimately, the more closely that we align research and community needs in PET/MR, the more efficiently can we reveal and move the field toward its ideal destination.

\section{DISCLOSURE}

No potential conflict of interest relevant to this article was reported.

\section{REFERENCES}

1. Martin O, Schaarschmidt BM, Kirchner J, et al. PET/MRI versus PET/CT for whole-body staging: results from a single-center observational study on 1,003 sequential examinations. J Nucl Med. 2020;61:1131-1136.

2. Karlberg AM, Saether O, Eikenes L, Goa PE. Quantitative comparison of PET performance-Siemens Biograph mCT and mMR. EJNMMI Phys. 2016;3:5.

3. Umutlu L, Beyer T, Grueneisen JS, et al. Whole-body $\left[{ }^{18} \mathrm{~F}\right]-\mathrm{FDG}-\mathrm{PET} / \mathrm{MRI}$ for oncology: a consensus recommendation. Nuklearmedizin. 2019;58:68-76.

4. Ehman EC, Johnson GB, Villanueva-Meyer JE, et al. PET/MRI: where might it replace PET/CT? J Magn Reson Imaging. 2017;46:1247-1262.

5. Rogers EM. Diffusion of Innovations. Free Press of Glencoe; 1962. 